- RAM, REV. ADM. MACKENZIE, V. 11, N. 6, Edição Especial • SÃO PAULO, SP • NOV./DEZ. 2010 • ISSN 1678-6971 • Submissão: $1^{\circ}$ out. 2009. Aceitação: 27 ago. 2010. Sistema de avaliação: às cegas dupla (double blind review). UNIVERSIDADE PRESBITERIANA MACKENZIE. Walter Bataglia (Ed.), p. 107-130.

\title{
SISTEMÁTICA MULTICRITÉRIO PARA PRIORIZAÇÃO DE EMBARQUES MARÍTIMOS
}

\section{PRIORITIZING MARITIME BOOKING CONFIRMATION USING MULTICRITERIA}

\section{DENISE LINDSTROM BANDEIRA}

Doutora em Administração pelo Programa de Pós-Graduação em Administração da Universidade Federal do Rio Grande do Sul (UFRGS).

Professora do Departamento de Ciências Administrativas da Universidade Federal do Rio Grande do Sul.

Rua Washington Luís, 855, sala 313, Centro - Porto Alegre - RS - Brasil - CEP 90010-460

E-mail: dlbandeira@ea.ufrgs.br

\section{JOÃO LUIZ BECKER}

Doutor em Administração pela Anderson Graduate School of Management da University of California, Los Angeles. Professor do Departamento de Ciências Administrativas da Universidade Federal do Rio Grande do Sul (UFRGS).

Rua Washington Luís, 855, sala 313, Centro - Porto Alegre - RS - Brasil - CEP 90010-460

E-mail: jlbecker@ea.ufrgs.br

\section{AMANDA KRUSE ROCHA}

Especialista em Gestão de Operações Logísticas pelo Programa de Pós-Graduação Lato Sensu da Universidade Federal do Rio Grande do Sul (UFRGS).

Rua Sete de Setembro, 745, $7^{\circ}$ andar, Centro - Porto Alegre - RS - Brasil - CEP 90010-190

E-mail: akrocha@terra.com.br 


\section{RESUMO}

O objetivo do artigo é apresentar o processo de decisão em grupo realizado junto a executivos de uma agência marítima com operação no Sul do Brasil, no qual a técnica Processo de Análise Hierárquica (AHP) foi utilizada como suporte. A decisão envolveu a priorização de confirmações de reservas de embarque de cargas de exportação em um determinado instante, em situação de escassez - falta de contêineres vazios ou espaços nos navios disponíveis. O contexto do estudo foi o porto de Rio Grande, no Estado do Rio Grande do Sul. A escolha da organização e do porto foi feita por conveniência. Utilizou-se a metodologia de resolução de problemas da pesquisa operacional (formulação do problema, construção do modelo, solução do modelo, teste do modelo e avaliação da solução e avaliação final) para gerar um modelo matemático para auxiliar os decisores na priorização de reservas de embarque de cargas. Dados secundários foram coletados da empresa estudada. Foram feitas reuniões para geração do modelo com a equipe comercial da empresa, composta por quatro funcionários e um gerente. O método mostrou-se apropriado para expandir a mente dos decisores, ao oportunizar a incorporação no processo decisório de outros fatores, além da visão meramente financeira, considerando fatores gerenciais e organizacionais. A validação do processo pelo grupo de executivos incluiu uma apreciação da qualidade da decisão tomada. Os executivos envolvidos foram unânimes ao afirmarem sua satisfação com os resultados obtidos a partir da utilização da técnica.

\section{PALAVRAS-CHAVE}

Tomada de decisão em grupo; AHP; Alocação de carga de exportação; Armazenagem em navios; Análise multicritério.

\section{ABSTRACT}

The aims of this article is to present the group decision-making process carried out with executives from a shipping agency operating in the south of Brazil, in 
which the Analytic Hierarchy Process (AHP) technique was used as support. The decision involved the prioritization of booking confirmations for the shipment of cargos for export at a particular moment in time, in a situation of scarcity - a lack of empty containers or space on the available vessels. The study context was the harbor of Rio Grande, in the State of Rio Grande do Sul. The choice of the organization and the harbor was done by convenience. It was used the methodology of problem solution of the operating research (problem formulation, model design, model solution, model testing and solution evaluation, and final evaluation) to generate a mathematical model for supporting decision makers on prioritizing booking confirmations for shipment of cargos. Secondary data were collected from the studied company. It was done meetings for generating the model with the commercial team of the company, composed by four employees and one manager. The method proved appropriate for broadening the mind of the decision makers as it facilitated the incorporation within the decision making process of other factors rather than the purely financial view, by taking into consideration managerial and organizational factors. The validation of the process by the group of executives included an assessment of the quality of the decision taken. The executives involved were unanimous in stating their satisfaction with the results obtained with the use of the technique.

\section{KEYWORDS}

Group decision-making; AHP; Allocation of export cargo; Storage on vessels; Multicriteria analysis.

\section{INTRODUÇÃO}

O transporte marítimo caracteriza-se por ser realizado em oceanos e mares, usando navios de grande porte, a fim de conduzir vários tipos de carga entre portos de um mesmo país ou de países diferentes. É realizado por armadores, pessoas jurídicas estabelecidas e registradas em um determinado país, com a finalidade de realizar transporte marítimo, local ou internacional, com a operação de navios, explorando determinadas rotas.

Ao transportar cargas de todos os tipos de um porto a outro, o armador tornase responsável pela carga que está transportando (KEEDI; MENDONÇA, 2000). Entretanto, o contato dos comerciantes com os armadores usualmente não é realizado de modo direto, sendo intermediado por agentes marítimos. A agência marítima é a empresa que representa o armador em alguma região ou mesmo 
em algum porto, atuando como seu mandatário mercantil, e serve de intermediária entre o armador e o comerciante, seja ele importador, seja exportador, controlando as operações de carga e descarga e angariando carga para o espaço disponível nos navios. A agência marítima representa, também, o armador junto a autoridades portuárias e governamentais.

Ao longo dos anos, com relação à movimentação de cargas no longo curso, vinculada ao comércio exterior, o Brasil tem sido predominantemente exportador, conforme demonstra o Gráfico I, a seguir.

\section{GRÁFICO I}

EVOLUÇÃO DA MOVIMENTAÇÃO DE CARGAS (COMÉRCIO EXTERIOR) NO LONGO CURSO, POR SENTIDO (I995-2007)

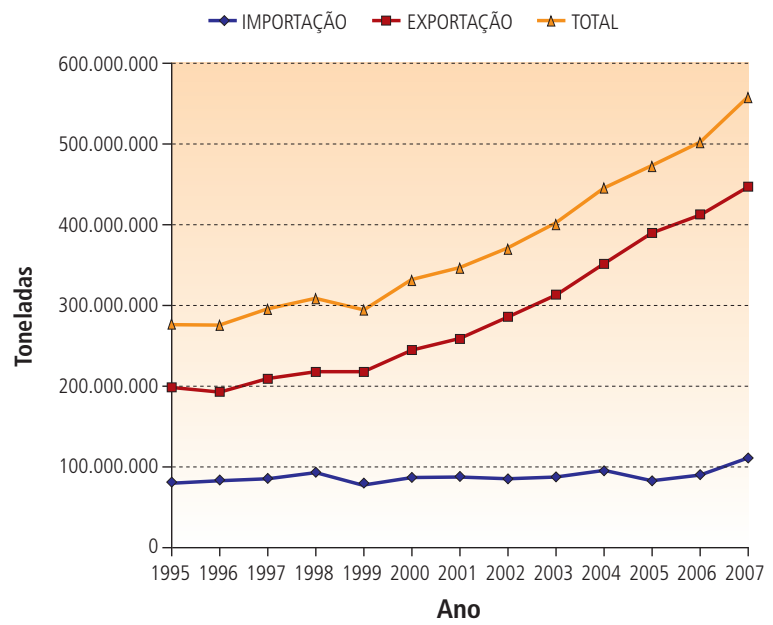

Fonte: Antaq (2007).

Isso é um reflexo das condições econômicas e do posicionamento do Brasil com relação às demais nações do mundo. Segundo a Organização Mundial do Comércio, em 2007, o Brasil foi o $24^{\circ}$ maior exportador mundial e o $28^{\circ}$ maior importador (WTO, 2009). As exportações são predominantemente commodities agrícolas e minerais $(50 \%)$ e manufaturados $(47,2 \%)$. O mesmo perfil ilustrado no Gráfico I se reproduz quando se analisa o volume de comércio exterior embarcado em contêineres.

Segundo dados da Agência Nacional de Transportes Aquaviários, no ano de 2007 foram movimentados 3.475.693 contêineres de 24 pés, na navegação de longo curso no Brasil (ANTAQ, 2007). A Tabela I apresenta a movimentação de contêineres em 2007 no Brasil e, em especial, do porto de Rio Grande (RS), local de atuação da empresa objeto do estudo de caso descrito neste artigo. 
TABELA I

MOVIMENTAÇÃO DE CONTÊINERES DE $20^{\prime}$ E 40', NOS PORTOS ORGANIZADOS E TERMINAIS DE USO PRIVATIVO, NA NAVEGAÇÃO DE LONGO CURSO, POR SENTIDO - 2007

\begin{tabular}{lcccccccc}
\hline & \multicolumn{3}{c}{ DESEMBARQUE } & \multicolumn{3}{c}{ EMBARQUE } \\
\cline { 2 - 9 } PORTO & \multicolumn{2}{c}{$20^{\prime}$} & \multicolumn{2}{c}{$40^{\prime}$} & $20^{\prime}$ & \multicolumn{2}{c}{$40^{\prime}$} \\
\cline { 2 - 9 } & CHEIO & VAZIO & CHEIO & VAZIO & CHEIO & VAZIO & CHEIO & VAZIO \\
\hline Rio Grande & 22.661 & 25.668 & 26.361 & 83.263 & 44.180 & 3.741 & 105.118 & 5.190 \\
\hline Brasil & 464.514 & 297.024 & 535.868 & 435.761 & 693.113 & 58.568 & 879.544 & 111.301 \\
\hline
\end{tabular}

Fonte: Antaq (2007).

Como se pode perceber, a movimentação de embarques (cerca de I,5 milhão de contêineres cheios em 2007) é muito superior à movimentação de desembarques (cerca de um milhão de contêineres cheios em 2007). Se essa desproporção de movimentação entre embarques e desembarques não é muito preocupante para cargas transportadas em navios graneleiros, ela se torna crítica quando se trata de movimentação por contêineres, gerando um severo desequilíbrio.

Contêineres vazios necessitarão ser transportados para corrigir o problema (cerca de 500 mil contêineres vazios em 2007), gerando custos adicionais, sem contrapartida de frete aos armadores.

A limitada capacidade dos navios e a relativa escassez de contêineres vazios dão origem a um problema de priorização no atendimento de solicitações de clientes por parte do agente marítimo, em uma dada janela temporal.

O objetivo deste artigo é apresentar um método de priorização do fechamento de reservas de embarque de cargas de exportação em um determinado instante, em situação de escassez - falta de contêineres vazios ou espaços nos navios disponíveis -, aplicando a técnica Processo de Análise Hierárquica (Analytic Hierarchy Process - AHP).

A intenção é demonstrar, na aplicação da técnica, a possibilidade de analisar critérios fora da visão meramente financeira, levando em consideração outros fatores, gerenciais e organizacionais, que possam melhorar a decisão. A utilização da técnica AHP tem permitido aos administradores a criação de cenários para situações existentes, que possam auxiliar na tomada de decisão. Essa aplicação em uma agência marítima tem por objetivo selecionar critérios que contribuam estrategicamente para avaliar as diversas demandas por espaço, possibilitando, assim, melhor priorização. 
O estudo limita-se ao porto de Rio Grande, no Rio Grande do Sul, por conveniência, especialmente no que se refere à disponibilidade de dados para efetuar as análises e entrevistar os gerentes responsáveis pela decisão. Posteriormente, o método desenvolvido poderá ser estendido para outras regiões.

$\mathrm{O}$ artigo se organiza da seguinte forma: a seção 2 apresenta a contextualização do estudo e a situação problemática; a seção 3 descreve a técnica de solução utilizada, apresentando o método na seção 4; a seção 5 traz o desenvolvimento do modelo; na seção 6 são analisados os resultados obtidos, e, na seção 7, fazem-se as considerações finais.

\section{CONTEXTUALIZAÇÃO DO ESTUdO}

\subsection{CARACTERIZAÇÃO DA EMPRESA}

A agência marítima do estudo é uma empresa estabelecida no Brasil desde I837, sendo a agência com maior cobertura nacional, ao longo da costa brasileira, atendendo aproximadamente a quarenta e cinco portos desde o porto de Rio Grande, no Rio Grande do Sul, até o porto de Manaus, no Estado do Amazonas. É responsável por I3\% da movimentação total de contêineres no Brasil, especializando-se no atendimento liner e tramp (navios de linhas regulares e de linhas não regulares, respectivamente). Em 2006, atendeu a 6.630 navios, obtendo receita líquida de US\$ I7,8 milhões com a prestação de serviços de agenciamento marítimo. Sua base de mais de 7.000 clientes contempla empresas de diversos setores, em todos os continentes.

O caso apresentado neste artigo focaliza o agenciamento de rotas regulares, a partir do porto de Rio Grande, oferecidas por um armador internacional que opera uma diversificada frota de navios para contêineres, automóveis, carga solta, petróleo, gás, carga geral e passageiros, totalizando mais de setecentas embarcações.

Os serviços prestados pelo armador a partir do porto de Rio Grande envolvem duas linhas: a East Coast South America - África - Ásia (CSW), em parceria com outros armadores; e a American Coast Express (ACX), alugando slots em navios de terceiros. A rota de exportação do serviço CSW é Buenos Aires - Montevidéu - Paranaguá - São Francisco do Sul - Santos - Rio de Janeiro - Cape Town - Port Elizabeth - Singapore - Hong Kong - Kobe - Yokohama - Nagoya - Busan - Shangai, com serviço semanal de feeder entre Buenos Aires - Montevidéu - Rio Grande (Figura I). A linha CSW opera com o total de II navios full containers com capacidade de 2.800 TEUs. 


\section{FIGURA I}

\section{ROTA DO SERVIÇO CSW}

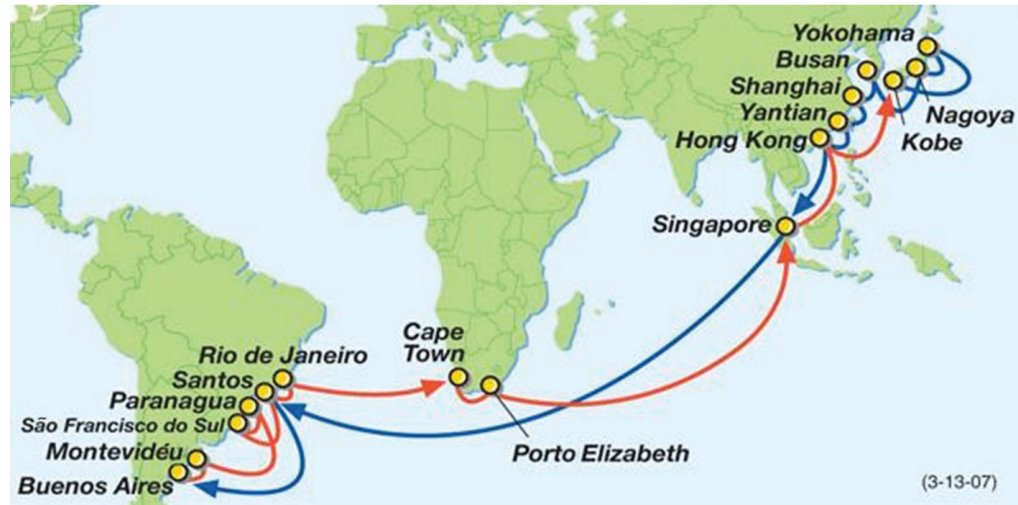

Fonte: Dados da pesquisa extraídos pelos autores do website do armador internacional.

Os tempos de trânsito, a partir do porto de Rio Grande para os destinos diretos do serviço CSW, são apresentados no Quadro I.

\section{QUADRO I}

TEMPO DE TRÂNSITO DO SERVIÇO CSW

\begin{tabular}{lc}
\hline & TEMPO DE TRÂNSITO DO SERVIÇO CSW A PARTIR DE RIO GRANDE \\
\hline CIDADE & DIAS \\
\hline Cape Town (África do Sul) & 21 \\
\hline Port Elizabeth (África do Sul) & 24 \\
\hline Singapore (Singapore) & 37 \\
\hline Hong Kong (Hong Kong) & 41 \\
\hline Kobe (Japão) & 46 \\
\hline Yokohama (Japão) & 47 \\
\hline Nagoya (Japão) & 48 \\
\hline Busan (Korea) & 50 \\
\hline Shanghai (China) & 52 \\
\hline Yantian (China) & 54 \\
\hline
\end{tabular}

Fonte: Dados da pesquisa extraídos pelos autores do website do armador internacional. 
A cobertura completa da linha ACX é: Buenos Aires - Rio Grande - São Francisco do Sul - Santos - Salvador - Norfolk - Philadelphia - New York - Baltimore - Buenos Aires (Figura 2).

\section{FIgURA 2}

\section{ROTA DO SERVIÇO ACX}

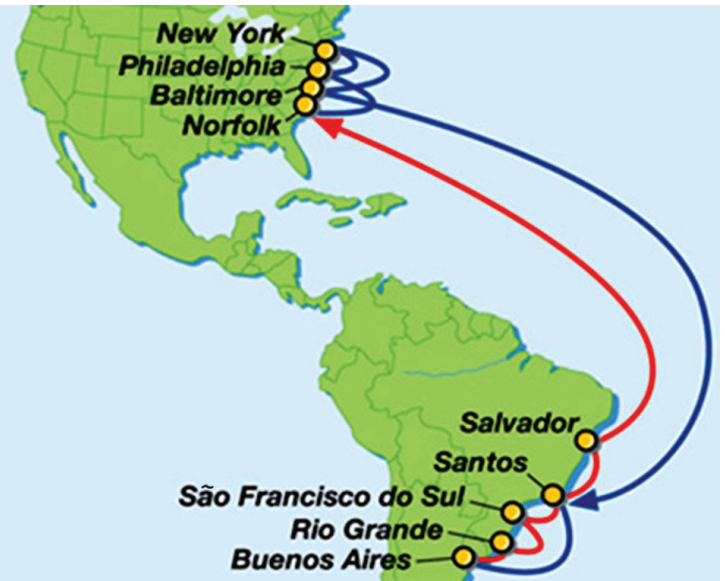

Fonte: Dados da pesquisa extraídos pelos autores do website do armador internacional.

Os tempos de trânsito, a partir do porto de Rio Grande para os destinos diretos do serviço ACX, são apresentados no Quadro 2.

\section{QUADRO 2}

TEMPO DE TRÂNSITO DO SERVIÇO AC

TEMPO DE TRÂNSITO DO SERVIÇO ACXA PARTIR DE RIO GRANDE

\begin{tabular}{lc}
\hline CIDADE & DIAS \\
\hline Norfolk & 18 \\
\hline Philadelphia & 20 \\
\hline New York & 21 \\
\hline Baltimore & 24
\end{tabular}

Fonte: Dados da pesquisa extraídos pelos autores do website do armador internacional. 


\subsection{SITUAÇÃO PROBLEMÁTICA}

Como já salientado, a balança comercial brasileira, particularmente a do Estado do Rio Grande do Sul, é marcadamente desbalanceada no sentido exportador. Como decorrência, o mercado de movimentação de cargas no porto de Rio Grande caracteriza-se pela relativa escassez de contêineres e de espaços nos navios porta-contêineres. Assim, um dos problemas recorrentes enfrentados pela agência marítima é a priorização de cargas de seus clientes nas escalas previstas. Cargas preteridas em uma determinada escala, por escassez de espaço, são programadas para a escala seguinte, gerando insatisfação nos clientes, aumentando o risco de a agência perdê-los para a concorrência. O problema é costumeiramente resolvido informalmente, ora a resolução pendendo para a alocação mais rentável, ora para a alocação de clientes mais fiéis, gerando insegurança nos gestores pela sensação de inconsistência de regras e procedimentos.

A alocação do armador no porto de Rio Grande é menor que a demanda dos clientes que utilizam esse porto para escoar seus embarques. Na linha CSW, a alocação é reduzida em razão de esse porto ser atendido via transbordo. O espaço limita-se, portanto, em decorrência do custo adicional, para mover as cargas de Rio Grande a Montevidéu, via serviço feeder. Na linha ACX, o espaço é limitado de acordo com o contrato de aluguel de espaço em navios de um terceiro armador.

Há também frequentes situações de indisponibilidade de contêineres de quarenta pés vazios em Rio Grande, causada pela dificuldade de reposicionamento de unidades vazias. As unidades vindas de importação para Rio Grande são, em sua maioria, de vinte pés.

Em marketing, a seleção de clientes é vista como o objetivo inicial da captura de clientes (customer acquisition), e a técnica de seleção mais utilizada é o modelo Recency, Frequency and Monetary value - RFM (BIJMOLT et al., 20IO). A captura de clientes, juntamente com o desenvolvimento de clientes e a retenção de clientes, constitui distinto estágio do ciclo de vida do cliente (Customer Lifetime Value - CLV), gerando o que é atualmente rotulado por engajamento do cliente (customer engagement).

Bijmolt et al. (2010) discutem o estado da arte de modelos para o engajamento de clientes. Diversos artigos são encontrados na literatura internacional, discutindo aspectos da seleção de clientes sob um ponto de vista mercadológico. Por exemplo, Venkatesan, Kumar e Bohling (2007) abordam a seleção de clientes com maior potencial sob restrição orçamentária do departamento de marketing, utilizando teoria da decisão bayesiana para acomodar a incerteza inerente na predição da resposta dos clientes.

Na literatura de processos estocásticos, o problema de seleção de clientes é usualmente denominado problema de controle de admissão (admission control problem), tendo sido originalmente concebido, segundo Son e Ikuta (2007), por 
Heyman (I968). Son (2007) e Son e Ikuta (2007) selecionam pedidos de clientes que chegam sequencialmente a um sistema de produção, representando o problema como um sistema de serviço com um único servidor e sem fila de espera.

Mais próximo dos objetivos deste estudo, Barbarosoğlu e Yazgaç (2000) desenvolveram uma ferramenta de apoio à decisão para um fabricante de motores elétricos a fim de auxiliá-lo a alocar sua capacidade entre diferentes clientes, de forma a maximizar seu valor de negócio. A ferramenta usa a técnica AHP para representar os critérios identificados pelo fabricante e obter uma priorização entre os clientes. As prioridades foram então utilizadas para estabelecer cotas de suprimento para os clientes.

Neste artigo, será utilizada a técnica AHP como fio condutor de um processo de decisão em grupo.

\section{ANALYTIC HIERARCHY PROCESS (AHP)}

O Processo de Análise Hierárquica (Analytic Hierarchy Process - AHP) é uma técnica de análise de decisão e solução de problemas complexos, envolvendo múltiplos critérios, desenvolvida por Thomas L. Saaty em meados da década de I970 (GOLDEN; WASIL; HARKER, I989).

O AHP baseia-se na relativa facilidade que a mente humana possui de estabelecer comparações entre dois objetos (pairwise comparisons). Uma classificação de vários objetos de um grupo pode então ser obtida a partir da combinação de comparações emparelhadas entre todos os objetos do grupo. Evidentemente, a multiplicidade de comparações emparelhadas entre todos os objetos de um grupo gera inconsistências, interpretadas como erros do processo cognitivo. Erros são geralmente eliminados, tomando a média das observações.

A simplicidade do processo, permitindo avaliar fatores qualitativos e quantitativos, tangíveis ou intangíveis, aliada à fundamentação matemática da teoria de erros, traz muita flexibilidade à técnica. Combinada com técnicas de convergência de julgamentos de diferentes indivíduos, com diferentes pontos de vista ou experiência, torna-se uma poderosa ferramenta de apoio à decisão em grupo. $\mathrm{O}$ AHP é hoje uma das técnicas mais conhecidas e utilizadas internacionalmente.

A aplicação da técnica é dividida em duas fases: estruturação e avaliação. Estruturação refere-se ao desenvolvimento de uma estrutura hierárquica modelando as relações entre o objetivo de decisão, critérios que traduzem o objetivo, critérios e alternativas de decisão (ver Figura 3). Avaliação refere-se aos julgamentos de valor que deverão ser estabelecidos entre diferentes alternativas, em face de cada subcritério ou critério, entre os subcritérios de cada critério e entre diferentes critérios ante o objetivo. 


\section{FIGURA 3}

\section{MODELO HIERÁRQUICO UTILIZADO PELA TÉCNICA AHP}

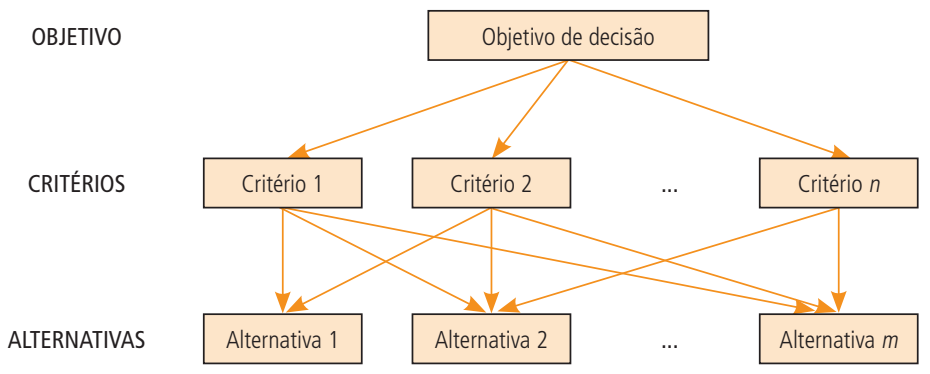

Fonte: Elaborada pelos autores.

Na medida em que o modelo hierárquico condiciona a fase de avaliação, é fundamental que ele esteja bem estabelecido entre os decisores, antes de se iniciar a segunda fase. Especificamente os decisores devem se assegurar de que a estrutura hierárquica seja completa (todas as alternativas e todos os critérios importantes para a decisão estão devidamente representados na hierarquia) e não redundante (não há sobreposição entre critérios ou alternativas). Adicionalmente, a técnica exige que os critérios sejam independentes entre si.

Saaty (I980) sugere que a fase de avaliação seja procedida por meio de julgamentos par a par, organizando os objetos sob julgamento (critérios, subcritérios, alternativas) em matrizes quadradas, cujos elementos indicam a importância de um objeto (linha) em relação a outro (coluna). O autor sugere também a utilização de uma escala de razão, representando a intensidade em importância ou preferência relativa de nove pontos, conforme apresentado no Quadro 3.

\section{QUADRO 3}

\section{ESCALA COMPARATIVA DE NOVE PONTOS}

\begin{tabular}{cl}
\hline VALORES NUMÉRICOS & DEFINIÇÃO \\
\hline 1 & Igualmente importante ou preferido \\
\hline 3 & Levemente mais importante ou preferido \\
\hline 5 & Medianamente mais importante ou preferido \\
\hline 7 & Fortemente mais importante ou preferido \\
\hline 9 & Extremamente mais importante ou preferido \\
\hline $2,4,6$ e 8 & Graus de importância ou preferência intermediários \\
\hline
\end{tabular}


A partir da organização das informações correspondentes aos julgamentos par a par em matrizes, estas são processadas com vistas à obtenção de uma ordenação dos objetos na escala de importância ou preferência, fornecendo sua prioridade relativa. Saaty (I980) propõe a utilização dos autovetores normalizados associados ao maior autovalor da matriz. Os autovetores representam a média de todas as possíveis maneiras de comparar os objetos dois a dois. Uma comparação entre o maior autovalor e a ordem da matriz produz uma interessante medida de inconsistência (consistency index - CI) dos julgamentos representados na matriz. Uma comparação desse autovalor com medidas de consistência de matrizes de mesma ordem geradas aleatoriamente produz uma medida denominada razão de consistência (RC), utilizada para aferir a qualidade geral do processo de julgamento. Valores de RC menores ou iguais a O,I são considerados aceitáveis. Valores superiores a O,I exigem a atenção dos avaliadores no sentido de reduzir suas inconsistências de julgamento, revisando tais valores devidamente.

Vaidya e Kumar (2006) e Ho (2008) apresentam abrangentes revisões de literatura com aplicações internacionais da técnica AHP em processos de tomada de decisão. No Brasil, encontram-se aplicações nas áreas de finanças (FREZATTI et al., 2007; MATOS; MOURA, 2003), gestão estratégica (MACEDO-SOARES; FIGUEIRA, 2007; TÁLAMO; CARVALHO, 2004), prospecção mineral (CUNHA; OLIVEIRA; SILVA, 200I), gestão de produção e logística (IAÑEZ; CUNHA, 2006; LAURINDO et al., 2002; RAFAELI; MÜLLER, 2007; ROSA; SELLITTO; MENDES, 2006; SELLITTO; MENDES, 2006; SELLITTO; WALTER, 2006; TORTORELLA; FOGLIATTO, 2008), prevenção de falhas (AGUIAR; SALOMON, 2007), marketing (CRUZ JUNIOR; CARVALHO, 2003), agronegócio (BARROS; MOREIRA; RUDORFF, 2007), meio ambiente (LUZ; SELLITTO; GOMES, 2006).

Mais de uma centena de teses e dissertações foram desenvolvidas utilizando AHP (CAPES, 2009). Vilas Boas (2005) apresenta uma boa revisão da técnica.

\section{MÉTODO}

Este estudo usou como base a metodologia de resolução de problemas desenvolvida no âmbito da pesquisa operacional (ACKOFF; SASIENI, I968; WAGNER, I986), com a proposta de empregar um modelo matemático para auxiliar a priorização de reservas de embarque de cargas por uma agência marítima. Foram utilizadas as etapas clássicas de um estudo de pesquisa operacional: formulação do problema (como priorizar reservas de embarque fora da visão estrita de análise financeira); construção do modelo (ver seção 5.I); solução do modelo (método AHP, utilizando planilha eletrônica, ver seções 5.2 e 5.3); teste do modelo e avaliação da solução (validação de face com executivos da empresa, ver seção 5.4); e avaliação final (ver seção 6). 


\section{DESENVOLVIMENTO DO MODELO}

Esta seção descreve a construção do modelo de avaliação multicritério para priorizar o fechamento de reservas de embarque de cargas de exportação em um determinado instante, utilizando a técnica AHP.

\subsection{CONSTRUÇÃO DO MODELO HIERÁRQUICO}

A Figura 4 apresenta a árvore hierárquica desenvolvida no estudo.

\section{FIGURA 4}

ESTRUTURA HIERÁRQUICA DO PROBLEMA DE PRIORIZAÇÃO DE EMBARQUES

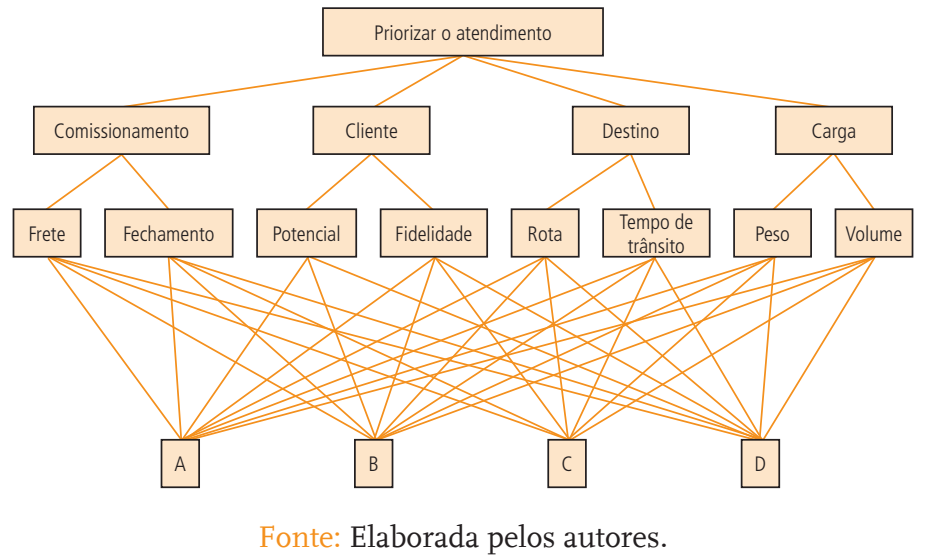

O objetivo, no primeiro nível, é definido pela questão: "Como priorizar o atendimento à demanda de exportação dos clientes da agência marítima?”. Os critérios e subcritérios, no segundo e terceiro níveis, respectivamente, foram obtidos de forma interativa, em reuniões sucessivas com a equipe comercial da filial de Porto Alegre da empresa, composta por quatro funcionários e um gerente.

Tendo por base o plano estratégico da empresa e suas metas comerciais, o grupo foi levado a refletir sobre quais critérios têm sido considerados significativos para a diferenciação entre as diversas solicitações dos clientes, no momento de fechamento de reservas. Quatro critérios emergiram do processo: comissionamento, cliente, destino e carga. Comissionamento refere-se a detalhes da carga propriamente dita, decomposto em dois subcritérios: valor monetário do frete e importância relativa do escritório responsável pelo fechamento. Cliente refere-se a aspectos valorizados pela empresa em seu relacionamento com os clientes, como 
potencial (futuro) e fidelidade (passado). Destino, por sua vez, refere-se a aspectos ligados ao traslado propriamente dito, decompondo-se em dois subcritérios: posicionamento competitivo da empresa relativamente aos seus concorrentes na rota escolhida pelo cliente e tempo de trânsito. Carga refere-se a características físicas do carregamento, decompondo-se em dois subcritérios: peso e volume.

As definições operacionais dos subcritérios (terceiro nível) são explicitadas a seguir:

- Frete: refere-se ao valor monetário do frete marítimo, expresso em moeda norte-americana (USD), estabelecido pelo armador e aceito pelo cliente.

- Fechamento: consideram-se o escritório responsável pela negociação junto ao cliente e a efetivação da confirmação de praça, pois a comissão recebida do armador deverá ser dividida entre os envolvidos (representante do porto de embarque e escritório mais próximo à região do cliente).

- Potencial: consideram-se o potencial do cliente no mercado, seu poder de influência e suas parcerias.

- Fidelidade: retrata o suporte dado pelo cliente à empresa em épocas anteriores.

- Rota: considera-se a rota da carga até o destino final, analisando a posição competitiva da empresa em relação aos seus concorrentes, como exclusividade ou interesse na rota. Os embarques poderão ser diretos ou atendidos via transbordo, considerando a atual situação de cada porto e os serviços feeders para os destinos finais.

- Tempo de trânsito: refere-se ao tempo estimado (em dias) de trânsito desde a saída do navio no porto de embarque até a descarga da unidade no destino final.

- Peso: refere-se ao peso ( $\mathrm{t}$ ) da carga.

- Volume: refere-se ao volume envolvido por período (contêineres/mês).

O último nível, representando as alternativas de decisão, é formado pelas demandas de clientes por serviços da empresa, em um dado momento. Descreve-se a seguir o caso de uma determinada semana. Nessa semana, há dois clientes regulares e dois potenciais clientes que contataram a agência solicitando cotações de frete marítimo e reservas. As reservas de praça são confirmadas assim que a disponibilidade de espaço e de equipamento para a semana solicitada for liberada.

Apresenta-se, a seguir, uma breve descrição de cada alternativa.

- Alternativa A: O cliente "A" é um agente de carga com especialização no segmento de couro, atuando no mercado desde I989, sendo um cliente regular 
da agência marítima. Possui uma posição de destaque no ramo e conta com agentes em diversos países. A solicitação de fechamento de reserva de praça decorre de cotação solicitada pelo cliente para embarque de aproximadamente seis toneladas de couro, em um contêiner de 40 pés, a partir do porto de Rio Grande até o destino final Cape Town, na África do Sul. O volume total contratado entre exportador e importador é de um contêiner por mês, durante o período de um ano. O frete aplicado é USD 2.500,00 por contêiner, mais taxas de combustível, armazenagem e liberação. As taxas adicionais serão desconsideradas neste estudo. Atualmente, a empresa representa o único armador a atender a Cape Town por meio de escalas diretas, e o espaço nos navios reservados para a África está comprometido em decorrência da grande demanda.

- Alternativa B: O cliente "B" é uma indústria do interior de São Paulo, que atende principalmente ao mercado interno, não tendo contratos firmados para embarques futuros. Chega à empresa fruto de uma nova conta desenvolvida pela filial de São Paulo. Portanto, se efetivado o embarque, a comissão deverá ser dividida entre a filial de Porto Alegre e a filial de São Paulo. A solicitação de reserva é de um contêiner contendo pneus, pesando aproximadamente 26 toneladas. O frete está acordado em USD 2.800,00 para embarque em Rio Grande com destino a Shimizu, no Japão, via Cingapura, com tempo de trânsito estimado em 42 dias. Cargas com destino ao Japão são estimuladas pelo armador, pois, apesar do longo tempo de trânsito, unidades vazias são rapidamente realocadas em outras linhas do próprio armador.

- Alternativa C: A empresa "C" iniciou suas atividades de assessoria aduaneira e logística em i984 e vem expandindo sua atuação no comércio exterior brasileiro. Apesar de não ter comprometimento de embarques com os armadores que a agência marítima representa, esse cliente tem boa relação com a agência. $\mathrm{O}$ frete foi negociado pelo importador junto ao armador nos Estados Unidos, com valor acordado de USD I.900,00, e está embutido em contrato de serviço mundial, o qual prevê uma média de embarque de um contêiner por semana. A presente reserva trata de um contêiner contendo móveis, com aproximadamente 20 toneladas, devendo embarcar de Rio Grande à Filadélfia, nos Estados Unidos.

- Alternativa D: Este agente de carga abriu recentemente escritório em Porto Alegre e, apesar de não ter grande presença no mercado gaúcho, está apostando em bons profissionais para firmar parcerias com tradicionais exportadores da Região Sul. O cliente " $D$ " solicitou à agência marítima uma cotação para embarque de I4 toneladas de tabaco de Rio Grande à cidade de Danville, no Estado de Kentucky, Estados Unidos. O serviço a ser prestado 
pelo armador é de descarga do contêiner no porto de Norfolk e transporte de caminhão até a porta do importador. O tempo de trânsito total estimado é de aproximadamente 32 dias. O frete foi acertado em USD 2.300,00 por contêiner.

\subsection{MATRIZES DE COMPARAÇÃO}

A partir da hierarquia representativa do problema (Figura 4), foram realizadas comparações par a par dos objetos de cada nível hierárquico com relação a cada objeto de nível hierárquico imediatamente superior. Dados $n$ objetos em um determinado nível hierárquico, serão necessários $n(n-1) / 2$ julgamentos com relação a cada objeto de nível hierárquico imediatamente superior, gerando correspondentemente uma matriz de preferências para cada um desses objetos. Inicia-se pelo nível mais baixo na estrutura, correspondente ao nível das alternativas, seguindo pelo nível imediatamente superior (nesse caso, o nível dos subcritérios), continuando pelo nível imediatamente superior (nesse caso, o dos critérios), sucessivamente, até atingir o nível mais alto, correspondente ao objetivo da análise.

Para o preenchimento das diversas matrizes de comparação, foi realizada uma reunião da equipe comercial, buscando consenso e validade aos valores atribuídos. O MS Excel ${ }^{\circledR}$ foi utilizado para facilitar a comunicação entre os participantes e o devido registro dos resultados. Os resultados das comparações das alternativas com relação aos subcritérios são apresentados nas tabelas 2 a 9, a seguir.

TABELA 2

COMPARAÇÃO DAS ALTERNATIVAS COM RELAÇÃO AO SUBCRITÉRIO F

\begin{tabular}{ccccccc}
\multicolumn{7}{c}{ COMPARAÇÃO DAS ALTERNATIVAS COM RELAÇÃO } \\
\hline & A & B & C & D & PRIORIDADE RELATIVA \\
\hline A & 1 & $1 / 3$ & 5 & 2 & 0,233 \\
\hline B & 3 & 1 & 8 & 5 & 0,579 \\
\hline C & $1 / 5$ & $1 / 8$ & 1 & $1 / 4$ & 0,050 \\
\hline D & $1 / 2$ & $1 / 5$ & 4 & 1 & 0,139 \\
\hline & & & & & RC $=0,035$ \\
\hline
\end{tabular}

Fonte: Elaborada pelos autores. 


\section{TABELA 3}

COMPARAÇÃO DAS ALTERNATIVAS COM RELAÇÃO AO SUBCRITÉRIO FECHAMENTO

\begin{tabular}{cccccc}
\hline & A & B & C & D & PRIORIDADE RELATIVA \\
\hline A & 1 & 6 & 1 & 1 & 0,316 \\
\hline B & $1 / 6$ & 1 & $1 / 6$ & $1 / 6$ & 0,053 \\
\hline C & 1 & 6 & 1 & 1 & 0,316 \\
\hline D & 1 & 6 & 1 & 1 & 0,316 \\
\hline
\end{tabular}

Fonte: Elaborada pelos autores.

\section{TABELA 4}

COMPARAÇÃO DAS ALTERNATIVAS COM RELAÇÃO AO SUBCRITÉRIO POTENCIAL DO CLIENTE

\begin{tabular}{cccccc}
\hline & A & B & C & D & PRIORIDADE RELATIVA \\
\hline A & 1 & 9 & 3 & 5 & 0,559 \\
B & $1 / 9$ & 1 & $1 / 8$ & $1 / 4$ & 0,040 \\
\hline C & $1 / 3$ & 8 & 1 & 4 & 0,294 \\
\hline D & $1 / 5$ & 4 & $1 / 4$ & 1 & 0,108 \\
\hline & & & & & $\mathbf{R C}=\mathbf{0 , 0 6 7}$ \\
\hline
\end{tabular}

Fonte: Elaborada pelos autores.

\section{TABELA 5}

COMPARAÇÃO DAS ALTERNATIVAS COM RELAÇÃO AO SUBCRITÉRIO FIDELIDADE DO CLIENTE

\begin{tabular}{cccccc}
\hline & A & B & C & D & PRIORIDADE RELATIVA \\
\hline A & 1 & 9 & 5 & 8 & 0,655 \\
\hline B & $1 / 9$ & 1 & $1 / 6$ & $1 / 2$ & 0,045 \\
\hline C & $1 / 5$ & 6 & 1 & 5 & 0,231 \\
\hline D & $1 / 8$ & 2 & $1 / 5$ & 1 & 0,068 \\
\hline & & & & & $\mathbf{R C}=\mathbf{0 , 0 8 0}$ \\
\hline
\end{tabular}

Fonte: Elaborada pelos autores. 


\section{TABELA 6}

COMPARAÇÃO DAS ALTERNATIVAS COM RELAÇÃO AO SUBCRITÉRIO ROTA

\begin{tabular}{cccccc}
\hline & A & B & C & D & PRIORIDADE RELATIVA \\
\hline A & 1 & $1 / 5$ & $1 / 8$ & $1 / 7$ & 0,041 \\
\hline B & 5 & 1 & $1 / 5$ & $1 / 4$ & 0,119 \\
\hline C & 8 & 5 & 1 & 3 & 0,550 \\
\hline D & 7 & 4 & $1 / 3$ & 1 & 0,290 \\
\hline
\end{tabular}

Fonte: Elaborada pelos autores.

\section{TABELA 7}

COMPARAÇÃO DAS ALTERNATIVAS COM RELAÇÃO AO SUBCRITÉRIO TEMPO DE TRÂNSITO

\begin{tabular}{cccccc}
\hline & A & B & C & D & PRIORIDADE RELATIVA \\
\hline A & 1 & 7 & $1 / 2$ & 4 & 0,326 \\
B & $1 / 7$ & 1 & $1 / 8$ & $1 / 6$ & 0,040 \\
\hline C & 2 & 8 & 1 & 5 & 0,503 \\
\hline D & $1 / 4$ & 6 & $1 / 5$ & 1 & 0,131 \\
\hline & & & & & $\mathbf{R C}=\mathbf{0 , 0 9 0}$ \\
\hline
\end{tabular}

Fonte: Elaborada pelos autores.

\section{TABELA 8}

COMPARAÇÃO DAS ALTERNATIVAS COM RELAÇÃO AO SUBCRITÉRIO PESO

\begin{tabular}{cccccc}
\hline & A & B & C & D & PRIORIDADE RELATIVA \\
\hline A & 1 & 7 & 5 & 3 & 0,551 \\
\hline B & $1 / 7$ & 1 & $1 / 4$ & $1 / 5$ & 0,051 \\
\hline C & $1 / 5$ & 4 & 1 & $1 / 4$ & 0,118 \\
D & $1 / 3$ & 5 & 4 & 1 & 0,281 \\
\hline & & & & & $\mathbf{R C}=\mathbf{0 , 0 9 1}$ \\
\hline
\end{tabular}

Fonte: Elaborada pelos autores. 


\section{TABELA 9}

COMPARAÇÃO DAS ALTERNATIVAS COM RELAÇÃO AO SUBCRITÉRIO VOLUME

\begin{tabular}{cccccc}
\hline & A & B & C & D & PRIORIDADE RELATIVA \\
\hline A & 1 & 9 & $1 / 4$ & 9 & 0,296 \\
\hline B & $1 / 9$ & 1 & $1 / 9$ & 1 & 0,045 \\
\hline C & 4 & 9 & 1 & 9 & 0,614 \\
\hline D & $1 / 9$ & 1 & $1 / 9$ & 1 & 0,045 \\
\hline
\end{tabular}

Fonte: Elaborada pelos autores.

No caso em estudo, cada um dos quatro critérios subdivide-se em apenas dois subcritérios. Portanto, a determinação das prioridades entre os subcritérios com relação a cada critério é mais facilmente obtida de modo direto, sem necessidade de elaboração de matrizes de comparação. O Quadro 4 apresenta o sentimento do grupo de executivos encarregados da avaliação.

\section{QUADRO 4}

PRIORIDADE RELATIVA ENTRE SUBCRITÉRIOS COM RELAÇÃO A CADA CRITÉRIO

\begin{tabular}{llc}
\hline CRITÉRIOS & \multicolumn{1}{l}{ SUBCRITÉRIOS } & PRIORIDADE RELATIVA \\
\hline \multirow{2}{*}{ Comissionamento } & Frete & 0,6 \\
\cline { 2 - 3 } & Fechamento & 0,4 \\
\multirow{2}{*}{ Cliente } & Potencial & 0,3 \\
\cline { 2 - 3 } & Fidelidade & 0,7 \\
\hline \multirow{2}{*}{ Destino } & Rota & 0,6 \\
\cline { 2 - 3 } & Tempo de trânsito & 0,4 \\
\hline \multirow{2}{*}{ Carga } & Peso & 0,4 \\
\cline { 2 - 3 } & Volume & 0,6 \\
\hline
\end{tabular}

Fonte: Elaborado pelos autores.

Finalmente, os resultados da comparação entre os critérios com relação ao objetivo da análise são apresentados na Tabela ıo. 


\section{TABELA IO}

COMPARAÇÃO DOS CRITÉRIOS COM RELAÇÃOAO OBJETIVO

\begin{tabular}{lccccc}
\hline & COMISSIONAMENTO & CLIENTE & DESTINO & CARGA & PRIORIDADE \\
\hline Comissionamento & 1 & 7 & 5 & 4 & 0,594 \\
\hline Cliente & $1 / 7$ & 1 & $1 / 5$ & $1 / 5$ & 0,049 \\
\hline Destino & $1 / 5$ & 5 & 1 & $1 / 2$ & 0,145 \\
Carga & $1 / 4$ & 5 & 2 & 1 & 0,212 \\
\hline & & & & & $\mathbf{R C}=\mathbf{0}, \mathbf{0 8 1}$ \\
\hline
\end{tabular}

Fonte: Elaborada pelos autores.

\subsection{VERIFICAÇÃO DE CONSISTÊNCIA}

Na seção anterior, foram apresentados os resultados finais consensuais do grupo de trabalho. De fato, várias reuniões foram realizadas visando avaliar a consistência interna das comparações realizadas. Inicialmente, as matrizes de comparação das alternativas com relação aos subcritérios frete, potencial do cliente, tempo de trânsito e volume apresentaram-se inconsistentes (RC superiores a o,I). Confrontado com tais inconsistências, o grupo aprofundou a análise, revisando suas avaliações e obtendo finalmente um modelo consistente.

\subsection{PRIORIZAÇÃO DAS ALTERNATIVAS}

A Tabela Iı apresenta o resultado final do modelo de priorização.

\section{TABELA II}

PRIORIZAÇÃO DAS ALTERNATIVAS

\begin{tabular}{cccccc}
\hline & COMISSIONAMENTO & CLIENTE & DESTINO & CARGA & \multirow{2}{*}{ COMPOSIÇÃO } \\
\cline { 2 - 5 } & $(0,594)$ & $(0,049)$ & $(0,145)$ & $(0,212)$ & \\
\hline A & 0,266 & 0,626 & 0,155 & 0,398 & $\mathbf{0 , 2 9 5}$ \\
\hline B & 0,368 & 0,044 & 0,088 & 0,047 & $\mathbf{0 , 2 4 4}$ \\
\cline { 1 - 2 } C & 0,156 & 0,250 & 0,531 & 0,416 & $\mathbf{0 , 2 7 0}$ \\
\hline D & 0,210 & 0,080 & 0,226 & 0,139 & $\mathbf{0 , 1 9 1}$ \\
\hline RC & $\mathbf{0 , 0 2 1}$ & $\mathbf{0 , 0 7 6}$ & $\mathbf{0 , 0 9 5}$ & $\mathbf{0 , 0 9 2}$ & $\mathbf{0 , 0 4 9}$ \\
\hline
\end{tabular}

Fonte: Elaborada pelos autores. 


\section{ANÁLISE DOS RESULTAdOS}

O resultado da priorização das alternativas obtida por meio da aplicação da técnica AHP, refletindo a escolha do grupo comercial, apresenta como primeira opção o atendimento ao cliente A, seguido pelos clientes C, B e D.

Uma comparação dos pesos relativos aos critérios gerais demonstra claramente que a empresa está focada no atendimento aos clientes com o melhor nível de comissionamento (0,594 contra O,2I2; O,I45 e O,I49). O posicionamento justifica-se na medida em que a empresa é remunerada por comissão sobre os fretes.

Buscando uma comparação com a sistemática usualmente utilizada pela agência, os participantes foram estimulados a refletir sobre a decisão em questão. Inicialmente confrontados com as quatro alternativas, antes da utilização da técnica AHP, a maioria dos participantes do processo de decisão escolheria o atendimento ao cliente A, enquanto somente uma pessoa do grupo escolheria atender o cliente C. À luz dos resultados alcançados com a técnica AHP, isso não chega a surpreender, na medida em que quase $60 \%$ do peso total entre os critérios recaem no critério de comissionamento (subdividido entre frete e fechamento), e as alternativas A e C são as mais vantajosas sob essa ótica.

Entretanto, os participantes foram unânimes em afirmar que a utilização da técnica esclareceu as diferenças de opinião no julgamento das alternativas, auxiliando-os a entender melhor a situação, o que facilitou a resolução de conflitos e a obtenção do desejado consenso. As maiores dificuldades registradas pelos executivos referem-se à comparação entre alternativas. Enquanto as comparações pareadas de critérios não apresentaram maiores problemas de inconsistência para os executivos, as comparações entre as alternativas apresentaram inconsistências iniciais. Tais inconsistências foram removidas durante o processo de barganha e acomodação das opiniões dos participantes.

A técnica revelou-se muito útil na resolução do problema, tendo sido especialmente destacadas sua facilidade e sua capacidade de tratar elementos quantitativos e qualitativos de forma interativa. A cada passagem pelo processo, na busca por resolução das inconsistências entre julgamentos, os participantes foram aprimorando sua compreensão individual e coletiva da difícil decisão enfrentada. Afinal, a situação de escassez de espaço nos navios que dá origem ao problema decisório resolvido pela priorização de embarque tem como consequência a negativa de embarque para alguns clientes, causando sua insatisfação. 


\section{CONSIDERAÇÕES FINAIS}

O modelo proposto neste artigo traz uma nova perspectiva ao processo de tomada de decisão na organização analisada. Ao auxiliar a decisão sobre ações operacionais (seleção de clientes e confirmações de reservas), com base nas diretrizes estratégicas da organização (agência), ampliou-se o campo de visão dos gestores. A técnica, por meio de suas análises e de seus resultados, traz tranquilidade ao tomador de decisão, na medida em que este percebe que a decisão está sendo tomada com o consenso tanto da equipe comercial como da gerência de toda a agência marítima.

A aplicação da técnica AHP neste estudo, caracterizada pela participação da equipe comercial e pela adoção de multicritérios, ricamente distinguindo as alternativas, permite a documentação do processo decisório de atendimento a clientes. Dessa forma, a indicação da alternativa mais adequada é realizada por meio de um embasamento formal com a validação da aplicação do modelo. As opiniões dos diferentes agentes envolvidos refletem-se nas análises por meio dos pesos dos critérios e dos indicadores atribuídos.

Sem o auxilio de uma técnica matemática, não seria possível avaliar os critérios e subcritérios conjuntamente e priorizar as alternativas sem arriscar inconsistências de julgamento, pelo fato de estes serem feitos intuitiva e desintegradamente. Obviamente, a técnica serve como apoio para a resolução de conflitos na alocação de recursos escassos e não substitui o decisor. Ao fornecer uma relação priorizada das alternativas, a técnica permite que a decisão seja tomada de forma mais confortável, uma vez que o resultado foi obtido por meio de vários níveis de análise e de forma consensual por uma equipe de especialistas.

Como sugestão para trabalhos futuros, o modelo proposto poderia ser estendido a outras situações de decisão importantes para a empresa. Por exemplo, a técnica poderia ser aplicada em situações de overbooking, quando a agência marítima precisa decidir cortar cargas de determinado navio.

\section{REFERÊNCIAS}

ACKOFF R. L.; SASIENI, M. Fundamentals of operations research. New York: John Wiley \& Sons, I968.

AGUIAR, D. C.; SALOMON, V. A. P. Avaliação da prevenção de falhas em processos utilizando métodos de tomada de decisão. Produção, v. I7, n. 3, p. 502-519, 2007.

ANTAQ. Anuário estatístico portuário (AEP). Brasília: Ministério dos Transportes, 2007. Disponível em: <http://www.antaq.gov.br/Portal/Anuarios/Portuariozoo7/Index.htm>. Acesso em: 23 abr. 2009 . 
BARBAROSOĞLU, G.; YAZGAÇ, T. A decision support model for customer value assessment and supply quota allocation. Production Planning and Control, v. II, n. 6, p. 608-6ı, 2000.

BARROS, M. A.; MOREIRA, M. A.; RUDORFF, B. F. T. Processo analítico hierárquico na identificação de áreas favoráveis ao agroecossistema cafeeiro em escala municipal. Pesquisa Agropecuária Brasileira, v. 42, n. I2, p. I769-1777, 2007.

BIJMOLT, T. H. A. et al. Analytics for customer engagement. Journal of Service Research, v. I3, n. 3, p. 34I-356, 2010.

CAPES. Banco de teses. Disponível em: <http://capes.gov.br/servicos/banco-de-teses >. Acesso em: 22 maio 2009 .

CRUZ JUNIOR, A. T.; CARVALHO, M. M. Obtenção da voz do consumidor: estudo de caso em um hotel ecológico. Produção, v. I3, n. 3, p. 88-100, 2003.

CUNHA, E. R. S. P.; OLIVEIRA, M. V.; SILVA, F. R. M. Utilização da técnica de processo analítico hierárquico (AHP) na avaliação da "favorabilidade" para a prospecção mineral de cromo na região de Pinheiros Altos, município de Piranga, MG, Brasil. REM: Revista da Escola de Minas, v. 54, n. 2, p. I27-I32, $200 \mathrm{I}$.

FREZATTI, F. et al. Análise do relacionamento entre a contabilidade gerencial e o processo de planejamento das organizações brasileiras. Revista de Administração Contemporânea, v. II, n. esp. 2, p. 33-54, 2007.

GOLDEN, B. L.; WASIL, E. A.; HARKER, P. T. The analytic hierarchy process: applications and studies. Berlim: Springer-Verlog, I989.

HEYMAN, D. P. Optimal operating policies for $M / G / I$ queuing systems. Operations Research, v. I6, n. 2, p. 362-382, I968.

HO, W. Integrated analytic hierarchy process and its applications - a literature review. European Journal of Operational Research, v. I86, n. I, p. 2II-228, 2008.

IAÑEZ, M. M.; CUNHA, C. B. Uma metodologia para a seleção de um provedor de serviços logísticos. Produção, v. I6, n. 3, p. 394-4I2, 2006.

KEEDI, S.; MENDONÇA, P. C. C. Transportes e seguros no comércio exterior. 2. ed. São Paulo: Aduaneiras, 2000.

LAURINDO, F. J. B. et al. Selecionando uma aplicação de tecnologia da informação com enfoque na eficácia: um estudo de caso de um sistema para PCP. Gestão e Produção, v. 9, n. 3, p. 377-396, 2002.

LUZ, S. O. C.; SELLITTO, M. A.; GOMES, L. P. Medição de desempenho ambiental baseada em método multicriterial de apoio à decisão: estudo de caso na indústria automotiva. Gestão e Produção, v. I3, n. 3, p. 557-570, 2006.

MACEDO-SOARES, T. D. L. A.; FIGUEIRA, L. A. P. A. Gestão estratégica da energia nucleoelétrica no Brasil: recursos e competências críticos para seu sucesso. Revista de Administração Contemporânea, v. II, n. esp. I, p. 55-76, 2007.

MATOS, D. M.; MOURA, H. J. Proposta de um modelo para determinação do custo de capital baseado em análise hierárquica. Revista de Administração Contemporânea, v. 7, n. 4, p. II9-I39, 2003.

RAFAELI, L.; MÜLLER, C. J. Estruturação de um índice consolidado de desempenho utilizando o AHP. Gestão e Produção, v. I4, n. 2, p. 363-377, 2007. 
ROSA, E. P. S.; SELLITTO, M. A.; MENDES, L. W. Avaliação multicriterial de desempenho e separação em aglomerados de fornecedores críticos de uma manufatura OKP. Produção, v. ı6, n. 3, p. 413-428, 2006.

SAATY, T. L. The analytic hierarchy process: planning, priority setting, resource allocation. New York: McGraw-Hill, ı980.

SELLITTO, M. A.; MENDES, L. W. Avaliação comparativa do desempenho de três cadeias de suprimentos em manufatura. Produção, v. ı6, n. 3, p. 552-568, 2006.

SELLITTO, M. A.; WALTER, C. Avaliação do desempenho de uma manufatura de equipamentos eletrônicos segundo critérios de competição. Produção, v. I6, n. I, p. 34-47, 2006.

SON, J. D. Customer selection problem with profit from a sideline. European Journal of Operational Research, v. I76, n. 2, p. I084-IIO2, 2007.

SON, J. D.; IKUTA, S. Customer selection problem with search cost, due date, sideline profit, and no waiting room. Asia-Pacific Journal of Operational Research, v. 24, n. 5, p. 647-666, 2007.

TÁLAMO, J. R.; CARVALHO, M. M. Seleção dos objetivos fundamentais de uma rede de cooperação empresarial. Gestão e Produção, v. II, n. 2, p. 239-250, 2004.

TORTORELLA, G. L.; FOGLIATTO, F. S. Planejamento sistemático de layout com apoio de análise de decisão multicritério. Produção, v. I8, n. 3, p. 609-624, 2008.

VAIDYA, O. S.; KUMAR, S. Analytic hierarchy process: an overview of applications. European Journal of Operational Research, v. I69, n. I, p. I-29, 2006.

VENKATESAN, R.; KUMAR, V.; BOHLING, T. Optimal customer relationship management using Bayesian decision theory: an application for customer selection. Journal of Marketing Research, v. 44, n. 4, p. 579-594, 2007.

VILAS BOAS, C. L. Método multicritérios de análise de decisão (MMAD) para as decisões relacionadas ao uso múltiplo de reservatórios: analytic hierarchy process (AHP). In: SIMPÓSIO BRASILEIRO DE RECURSOS HÍDRICOS, I6., 2005, João Pessoa. João Pessoa: ABRH, 2005.

WAGNER, H. M. Pesquisa operacional. Rio de Janeiro: Prentice-Hall do Brasil, I986.

WTO. International Trade Statistics 2008 - I. World trade developments in 2007. Geneva: World Trade Organization, 2009. Disponível em: <http://www.wto.org/english/res_e/statis_e/its2008_e/ itso8_world_trade_dev_e.pdf>. Acesso em: 25 maio 2009. 